\title{
Memoria 2017-2019 del Comité Científico de la Sociedad Chilena de Anestesia
}

\author{
2017-2019 Report of the Scientific Committeeof \\ the Chilean Society of Anesthesia
}

Héctor J. Lacassie ${ }^{1}$

I Comité Científico de la Sociedad Chilena de Anestesia (SACH) ha sido una unidad inherente a la razón de ser de la Sociedad desde sus inicios que, si bien no tenía una constitución formal, su funcionamiento ha sido fundamental para el desarrollo del quehacer científico y educacional de la Sociedad.

El funcionamiento del Comité se ha ido consolidando progresivamente hasta el año 2017 en que se establecieron los estatutos formales. En la estructura organizacional quedó definido que el directorio del Comité Científico depende del presidente y del Directorio de la SACH y que el nuevo directorio aumenta a un número de trece personas. Se establece que los tres pilares fundamentales de funcionamiento son: Investigación, Difusión y la Revista Chilena de Anestesia.

El Comité Científico es un ente dependiente y de confianza del Directorio de la Sociedad Chilena de Anestesia, aunque autónomo en su conformación. De acuerdo al ámbito de su competencia, sus labores y decisiones tienen relación con la promoción de la investigación científica de buena calidad en la especialidad y en la difusión del quehacer científico en la cual la Sociedad Chilena de Anestesia esté involucrada, además de colaborar en las actividades asignadas por el directorio a propósito del ámbito de sus funciones. Se establece que la visión del Comité Científico es: "Ser un Comité Científico en Anestesiología referente en América Latina, por su capacidad de emitir juicios con autonomía, transparencia y rigurosidad científica". Y a su vez, la misión es: "Representar a la Sociedad Chilena de Anestesia como su voz científica, en forma independiente, en todos los aspectos que esto involucre".

Se aprueban los nuevos estatutos del Comité Científico (marzo de 2017) y se da inicio al Comité en su período 2017-2019 formalmente donde se plantean los siguientes objetivos para su gestión: 1) preocuparse por el desarrollo científico de la especialidad; 2) promover la investigación de buena calidad y 3) promover la difusión del quehacer científico en el cual la Sociedad Chilena de Anestesia esté involucrada.

Una de las primeras actividades fue reestructurar y darle prioridad al Concurso de Investigación anual de la Sociedad. En 2017 se presentaron nueve trabajos y se eligieron dos. Los trabajos ganadores fueron: 1) "Estudio farmacocinético de levobupivacaína con epinefrina en bloqueo de transverso abdominal (TAP) para analgesia post cesárea". Autor principal: Héctor J. Lacassie, Pontificia Universidad Católica de Chile; y 2) "Efecto de la Analgesia Inducida por Opioides en Marcadores Electroencefalográficos de Dolor Agudo". Autor principal: José I. Egaña, Universidad de Chile. Ambos autores recibieron el aporte de $\$ 1.500 .000$ para proceder con su estudio.

Los premios del Concurso de Temas Libres del $44^{\circ}$ Congreso Chileno de Anestesiología (2016) fueron

Profesor Asociado, División de Anestesiología, Facultad de Medicina, Pontificia Universidad Católica de Chile. Past president Comité Científico de la Sociedad Chilena de Anestesia.

Fecha de recepción: 10 de diciembre de 2019

Fecha de aceptación: 11 de diciembre de 2019

\section{ORCID}

https://orcid.org/0000-0001-5758-4113

Correspondencia:

Héctor J. Lacassie

Email: Lacassie@med.puc.cl 
entregados a sus respectivos ganadores (Dr. Fernando Altermatt (Pontificia Universidad Católica de Chile) y Dr. Sebastián Paredes (Pontificia Universidad Católica de Chile).

El Concurso de Temas Libres del $45^{\circ}$ Congreso Chileno de Anestesiología se abrió en septiembre de 2017, donde se hizo especial hincapié en respetar la normativa legal chilena para la investigación[1]-[3]. Se evaluaron por 14 jueces, realizando 4-5 evaluaciones/ trabajo con una pauta de evaluación elaborada por los miembros del Comité Científico. Se estableció que los 10 mejores trabajos se presentarían en forma oral y serían evaluados por 7 jueces, utilizando una pauta ad hoc. Se recibieron 64 trabajos y se aceptaron 52 . Ese año se modificó los estatutos del concurso, estableciéndose los siguientes premios para este concurso y los futuros:

1) Primer lugar Oral: Pasaje, inscripción y estadía en congreso ASA del año siguiente + Certificado.

2) $2^{\circ}$ lugar Oral: Inscripción y estadía en congreso chileno del año siguiente + Certificado.

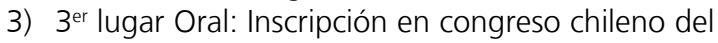
año siguiente + Certificado.

4) $1^{\mathrm{a}}$ Mención honrosa Póster: Inscripción en congreso chileno del año siguiente + Certificado.

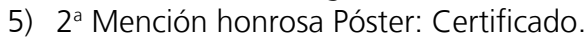

6) $3^{a}$ Mención honrosa Póster: Certificado.

El concurso evolucionó sin inconvenientes. Los autores ganadores fueron respectivamente: 1) Héctor J. Lacassie (Pontificia Universidad Católica de Chile): "Estudio farmacocinético de levobupivacaína con epinefrina en bloqueo de transverso abdominal (TAP) para analgesia post cesárea"; 2) Verónica Varas (Universidad de Chile) "Eficacia de la asociación de ketamina - magnesio en el dolor postoperatorio de Liposucción y Lipoabdominoplastía: Estudio randomizado doble ciego"; 3) Santiago Arze (Pontificia Universidad Católica de Chile) "Validación de Escalas de Satisfacción de Pacientes con la Anestesia: Informe Preliminar"; 4) José I. Egaña (Universidad de Chile) "Efectos de la analgesia farmacológica sobre marcadores electroencefalográficos de dolor agudo"; 5) Carlos Fuentes (Pontificia Universidad Católica de Chile) "Poder espectral y Saturación de Banda Alfa como predictores de pérdida de respuesta al comando verbal en adultos mayores de 65 años durante inducción lenta con propofol: Estudio de prueba diagnóstica" y 6) Alejandro González (Pontificia Universidad Católica de Chile) "Impacto de la educación preoperatoria estructurada sobre el nivel de ansiedad preoperatoria de pacientes pediátricos y de sus padres".

Otras actividades en las que estuvo involucrado el
Comité Científico fueron las reuniones científicas de la Sociedad. Se realizaron reuniones en los meses de marzo, mayo, junio y septiembre de 2017, con muy buena convocatoria y excelentes expositores. Se acordó que las reuniones fueran filmadas y subidas a la página web. La primera fue "Manejo del dolor agudo perioperatorio en niños" a cargo de Dras. María Isabel Pérez, Carla Hudson B. y Liliana Ramos. Presentaron su experiencia en el desarrollo e implementación de guías y normas de manejo del dolor agudo periopertorio en niños en el hospital Roberto del Río. En mayo se presentó la conferencia "Neurocience principles and case studies" que fue impartida por el invitado especial. Dr. Patrick Purdon de la Universidad de Harvard. En junio la reunión fue un Pro Con de la técnica analgésica para trabajo de parto: una visión desde una clínica privada y otra desde un hospital público. Fue una discusión académica en que los participantes defendieron la técnica que habitualmente hacen y cuestionaron la técnica alternativa del contrincante, basados en los mejores argumentos posibles. Los invitados fueron los Drs. Jorge Aguilera (Clínica Las Condes) y Juan Morales (Hospital La Florida/Clínica Alemana de Santiago). En septiembre la reunión estuvo a cargo del Comité de Anestesia Regional, donde presentaron los Drs. Julián Aliste (Universidad de Chile/Clínica Las Condes) con el tema "Bloqueos interfasciales: ESP y cuadrado lumbar", seguido del Dr. Pablo Miranda (Pontificia Universidad Católica de Chile) con la revisión: Recomendaciones ASRA sobre complicaciones neurológicas asociadas a anestesia regional".

Ese año se abrió la página web del Comité Científico como micrositio dentro de la página web de la SACH (https://www.sachile.cl/comites/comite-cientifi$\mathrm{CO}$.

En 2018 correspondió cambiar a 25\% de los directores (Drs. Gianpaolo Volpato y Alejandro Recart), además de reemplazar al Drs. Guillermo Lema y Juan Cristóbal Pedemonte, este último por estar en el extranjero realizando una estadía de perfeccionamiento. En tales condiciones, fueron propuestos y aceptados por la asamblea los Drs.: Julián Aliste (Universidad de Chile/Clínica Las Condes), René de la Fuente (Pontificia Universidad Católica de Chile), Fernando Aranda (Universidad de Valparaíso) y Juan Pablo Álvarez (Clínica Las Condes) (Tabla 1).

Para el Concurso de Investigación 2018 se presentaron cinco trabajos y se eligieron dos, de acuerdo a la normativa. Los trabajos ganadores fueron: 1) Julián Aliste (Universidad de Chile/Clínica Las Condes): “Una Comparación Randomizada Entre Dexametasona y Dexmedetomidina Como Coadyuvantes Para el Bloqueo Infraclavicular de Plexo Braquial Guiado por 


\begin{tabular}{llll} 
& & \multicolumn{2}{c}{ Tabla 1. Comité Científico SACH 2017-2020 } \\
\hline $\mathbf{N}^{\circ}$ & Nombre & Apellido & Centro \\
1 & Fernando & Altermatt & Pontificia Universidad Católica de Chile \\
2 & Francisca & Bernucci & Universidad de los Andes/Clínica Dávila \\
3 & Ma Carolina & Cabrera & Universidad de Valparaíso/Hospital FACH \\
4 & Esperanza & Carrasco & Clínica Alemana \\
5 & José lgnacio & Egaña** & Universidad de Chile \\
6 & Héctor & Lacassie* & Pontificia Universidad Católica de Chile \\
7 & Waldo & Merino & Universidad de la Frontera \\
8 & Antonello & Penna & Universidad de Chile \\
9 & David & Torres** & Universidad de los Andes/Clínica Santa María \\
10 & Julián & Aliste & Universidad de Chile/Clínica Las Condes \\
11 & René & De la Fuente & Pontificia Universidad Católica de Chile \\
12 & Fernando & Aranda & Universidad de Valparaíso \\
13 & Juan Pablo & Álvarez & Clínica Las Condes \\
14 & Guillermo & Lema & Pontificia Universidad Católica de Chile \\
15 & Alejandro & Recart*** & Clínica Alemana/Universidad del Desarrollo \\
16 & Gian Paolo & Volpato & Universidad de Chile \\
17 & Juan Cristóbal & Pedemonte & Pontificia Universidad Católica de Chile \\
\hline$*$ Presidente; & & \\
\hline
\end{tabular}

*Presidente; **Vicepresidente; ***Secretario; ****Past President.

Ultrasonografía" y 2) Waldo Merino (Universidad de la Frontera): "Precisión, confiabilidad y validez procedimental de la ecografía transtorácica en el posicionamiento y extensión del catéter venoso central en comparación con la radiografía de tórax y fórmula por talla". Ambos autores recibieron el aporte de $\$ 1.500 .000$ para proceder con su estudio.

Se recibió el informe de avance final del concurso 2016 y del avance anual del concurso de investigación 2017, permitiendo seguir con el proceso establecido.

El Concurso de Temas Libres del $46^{\circ}$ Congreso Chileno de Anestesiología se abrió el 27 de agosto de 2018. Para ese año se estableció que los quince mejores trabajos se presentarían en forma oral en el congreso. Se recibieron 34 trabajos y se aceptaron 32. Llamó la atención la disminución en casi $50 \%$ con respecto a los años previos en el número de trabajos enviados. El concurso evolucionó sin inconvenientes. Los autores ganadores fueron los Drs.: 1) Rodrigo Gutiérrez (Universidad de Chile): "Una menor razón alfa/delta en la señal electroencefalográfica intraoperatoria se asocia con un menor rendimiento cognitivo preoperatorio en pacientes mayores de 60 años"; 2) Sebastián Paredes (Pontificia Universidad Católica de Chile): "Biomarcadores en cirugía de aneurisma intra- craneales no rotos: estudio piloto" y 3) Rubén Heredia (Universidad de Santander): "Evaluación Prequirúrgica de Contenido Gástrico por Ultrasonido en Cirugía de Urgencias". En formato Póster los ganadores fueron: 1) Gabriela Fariña (Universidad de los Andes - Clínica Santa María) "Bloqueo ecoguiado de los ramos intercostales anteriores para manejo del dolor en cirugía cardiaca"; 2) Dagoberto Ojeda (Universidad de los Andes - Clínica Dávila) "Duración efectiva y factores determinantes de la analgesia con morfina intratecal en prótesis de cadera" y 3) María Carolina Cabrera (Universidad de Valparaíso) "Evaluación no invasiva de frecuencia respiratoria con dispositivo Dalí".

Con respecto a las reuniones científicas, se realizaron cuatro: en los meses de marzo, mayo, julio y septiembre de 2018, con muy buena convocatoria y excelentes expositores. En marzo se presentaron los trabajos ganadores del concurso de Temas Libres 2017. A partir de la reunión de mayo, las reuniones fueron filmadas y subidas a la página web, que han tenido muy buena recepción por parte de los socios. La reunión de mayo estuvo a cargo de los Drs. Gustavo Galleguillos y Rodrigo López (Pontificia Universidad Católica de Chile) que presentaron el tema "NIRS fuera de pabellón de cirugía cardíaca". En esa ocasión 
se estrenó una nueva sede para las reuniones clínicas que fue en el auditorio científico Mauricio Wainer de la Clínica Las Condes. La reunión de julio se presentó el manual "Ventrículo derecho y anestesia" de la Dra. María Carolina Cabrera, seguido del Pro Con "Bloqueos continuos para analgesia en cirugía artroscópica de hombro: ¿justificada o no?" a cargo de los Drs. Fernando Altermatt (Pontificia Universidad Católica de Chile) y Daniela Bravo (Universidad de Chile). En la reunión de septiembre se presentó el tema "Cardiomiopatía del embarazo. ¿Realidad en Chile?" por la Dra. Javiera Vargas (Clínica Alemana de Santiago/ Hospital Barros Luco). Hubo dos reuniones más, a cargo del Directorio de la Sociedad (Concepción) y otra del Comité de Pediatría (agosto de 2018).

En el micrositio dentro de la página web de la sociedad se publicó la "Infografía de la Investigación" (https://www.sachile.cl/comites/comite-cientifico/ infografia-investigacion), que fue pensada como un repositorio de temas atingentes a la investigación.

En 2019 el Concurso de Investigación se abrió en marzo de 2019. Se presentaron siete trabajos y se eligieron dos, de acuerdo a la normativa. Los trabajos ganadores fueron: "Validación Inicial del Poder Relativo de Banda Alfa como Biomarcador del Riesgo de Desarrollar Estados Confusionales Agudos Postoperatorios. Un estudio piloto" Autor principal: Felipe Maldonado, Universidad de Chile; e "Influencia del envejecimiento en la dosificación perioperatoria de metadona". Autora principal: Francisca Elgueta, Pontificia Universidad Católica de Chile. Ambos autores recibieron el aporte de $\$ 1.500 .000$ para proceder con su estudio, en la reunión clínica de agosto de 2019.

Se recibió el informe de avance final del concurso 2017 y del avance anual del concurso de investigación 2018, de acuerdo a las directrices del concurso.

El concurso de investigación de la SACH ha sido muy exitoso y ha permitido el desarrollo de investigación nacional de buena calidad, la que ha sido difundida en prestigiosas revistas nacionales y extranjeras de la especialidad. Durante esta administración, los trabajos que han sido financiados y que han sido publicados son:

- (2016) Lacassie HJ, Rolle A, Cortínez LI, Solari S, Corvetto MA, Altermatt FR. Pharmacokinetics of levobupivacaine with epinephrine in transversus abdominis plane block for postoperative analgesia after Caesarean section. British Journal of Anaesthesia. 2018 Aug;121(2):469-75.[4].

- (2017) Penna, Antonello y cols. Injuria Renal Aguda Perioperatoria. Protocolo de Estudio para Determinar el Rol del Factor de Crecimiento Fibroblástico 23 como Biomarcador de Diagnóstico
Temprano. Rev Chil Anest (en prensa, 2020). - (2018) Aliste J, Layera S, Bravo D, Fernández D, Jara Á, García A, et al. Randomized comparison between perineural dexamethasone and dexmedetomidine for ultrasound-guided infraclavicular block. Reg Anesth Pain Med 2019; doi:10.1136/rapm-2019100680.[5].

A su vez, el desenlace de los primeros 12 trabajos enviados como Temas Libres para el año 2017 fue bastante exitoso, al tener 5 trabajos que fueron publicados en revistas con comité editorial. Los trabajos publicados fueron:

- Lacassie HJ, Rolle A, Cortínez LI, Solari S, Corvetto MA, Altermatt FR. Pharmacokinetics of levobupivacaine with epinephrine in transversus abdominis plane block for postoperative analgesia after Caesarean section. British Journal of Anaesthesia. 2018 Aug;121(2): 469-75.[4].

- de la Fuente R, Fuentes R, Muñoz-Gama J, Riquelme A, Altermatt F, Pedemonte J, Corvetto M, Sepúlveda M. Control-Flow Analysis of Procedural Skills Competencies in Medical Training through Process Mining. Aceptado para publicación Postgraduate Medical Journal 10 de noviembre 2019. Manuscript id 2019-136802.

- Balkenhol M, Castillo A, Soto M, Feijoo M, Merino W, Balkenhol M, et al. Huella de carbono en el Hospital Base de Puerto Montt. Rev Med Chile 2018;146(12): 1384-9.[6].

- Fuentes R, Cortínez LI, Contreras V, Ibacache M, Anderson BJ. Propofol pharmacokinetic and pharmacodynamic profile and its electroencephalographic interaction with remifentanil in children. Paediatr Anaesth. 2018 Dec;28(12): 1078-86.[7].

- Aliste J, Bravo D, Finlayson RJ, De Q Tran. A randomized comparison between interscalene and combined infraclavicular-suprascapular blocks for arthroscopic shoulder surgery. J Can Anesth 2017 Dec $19: 1-8 .[8]$

Haciendo un catastro del desenlace de los 14 primeros trabajos enviados como Temas Libres para el año 2018 y de los 4 primeros lugares en la categoría póster, hasta el momento ha evolucionado bien, con 3 trabajos del formato oral que han sido publicados en revistas con comité editorial y uno de la categoría póster. Los trabajos publicados fueron:

- Bravo D, Aliste J, Layera S, Fernández D, Leurcharusmee $P$, Samerchua $A$, et al. A multicenter, randomized comparison between 2, 5, and $8 \mathrm{mg}$ of perineural dexamethasone for ultrasound-guided infraclavicular block. Regional Anesthesia and 
Pain Medicine. 2019 Jan;44(1):46-51.[9].

- Aguirre C., María Mercedes, et al. Registro de paro cardiorrespiratorio perioperatorio en un hospital universitario en el período 2006-2017. Rev Med Chile, 2019, vol.147, no.1, p.34-40. ISSN 0034-9887.[10].

- Gutiérrez R, Egaña Jl, Sáez I, Reyes F, Briceño C, Venegas M, et al. Intraoperative Low Alpha Power in the Electroencephalogram Is Associated With Postoperative Subsyndromal Delirium. Front Syst Neurosci. Frontiers; 2019; 13:56.[11].

- Contreras V, Fuentes R, Barros C, Casanueva O, Barrueto G. Implementación de un programa de reciclaje en pabellón. Rev Chil Anest. 2019; 48(2): 136-40.[12].

El Concurso de Temas Libres del año 2019 sufrió una variación con respecto a lo tradicional, ya que no se realizó el Congreso Chileno de Anestesia, sino que se juntó con CLASA en Buenos Aires. El concurso se realizó para los envíos de trabajos de autores locales y fue en paralelo a lo que ocurrió en CLASA. Se realizaron 10-13 evaluaciones/trabajo con una pauta de evaluación elaborada por los miembros del Comité Científico. Se recibieron 22 trabajos y los autores ganadores fueron los Drs.: $1^{\circ}$ ) Antonello Penna y cols. (Universidad de Chile). Efecto de dosis crecientes de propofol en la potencia relativa alfa hasta el burst suppression; $2^{\circ}$ ) David Torres y cols. (Universidad de los Andes/Clínica Santa María): Concordancia de monitores de temperatura para el postoperatorio inmediato. Cohorte observacional; y $3^{\circ}$ ) Daniela Bravo y cols. (Universidad de Chile): Analgesia en artroplastía de cadera: bloqueo de plexo lumbar versus fascia iliaca suprainguinal. Los premios fueron entregados en la reunión clínica de septiembre de 2019.

Con respecto a las reuniones científicas, se realizaron cuatro en los meses de marzo (presentación de los mejores tres Temas Libres del congreso 2018), julio ("Pro-Con dexmedetomidina vs dexametasona como coadyuvantes en bloqueos regionales" con los Drs. Jorge Castro (Clínica Alemana de Santiago) vs Sebastián Layera (Universidad de Chile)). Agosto: "Monitoreo y manejo del shock séptico basado en fisiología" Dr. Glenn Hernández (Pontificia Universidad Católica de Chile) y septiembre: "ERAS en cesárea", Dr. Alejandro Jankelevic (Universidad de Chile-Hospital $\mathrm{FACH}$ ), todas ellas con muy buena convocatoria y recepción de los asistentes. Las reuniones han continuado siendo filmadas y subidas a la página web, que tienen mucha visibilidad, con cerca de 100 personas conectadas por vez, constituyéndose en un beneficio más para los socios. Finalmente, hubo una reunión más, a cargo del Comité de Pediatría (noviembre de 2019).

Luego de este trienio, en 2020 corresponde cambiar al presidente (Dr. Héctor J. Lacassie) quien será reemplazado por el Dr. José Ignacio Egaña en la $1^{\text {a }}$ reunión científica del año, en marzo de 2020. Quedará a cargo del comité elegir nuevo vicepresidente. De acuerdo a los estatutos, en 2022 se deberá reemplazar $25 \%$ de los integrantes (3 directores).

Si bien participar en las actividades de la Sociedad implica un trabajo y empuje importante, es gratificante ver que las cosas finalmente se pueden hacer, teniendo un objetivo claro y un equipo de trabajo colaborador. Es deseable que las nuevas generaciones entiendan la importancia de pertenecer a nuestra Sociedad y de participar activamente para engrandecerla.

\section{Revista Chilena de Anestesia}

En la reunión de diciembre de 2016, el Directorio de la Sociedad eligió al Dr. Dagoberto Ojeda como Editor en jefe de la Revista Chilena de Anestesia. Se trabajó en conjunto con el Comité Científico para reflotar la revista luego de la última fallida dirección. Se publicaron 3 números, con la promesa de publicar el $4^{\circ}$ número del año en diciembre de 2017. El Editor decidió renunciar por motivos personales, a partir del 1 de enero de 2018. Se llamó a concurso para el cargo y la decisión para el nombramiento del nuevo Editor en Jefe sería en diciembre de 2017.

La nueva elección recayó en la Dra. María Carolina Cabrera para ejercer como Editor en Jefe de la Revista Chilena de Anestesia. Rápidamente la revista revivió gracias a una destacada y prolífica labor de la Editora. La revista actualmente está muy activa, publicándose 4 números al año. Se ha incorporado a varios metabuscadores, entre ellos la postulación y aceptación a Directory Open Access Journal (DOAJ) que es el buscador online más grande del mundo de revistas en formato electrónico y Google Scholar, que le han dado gran visibilidad nacional y latinoamericana, recibiendo trabajos cada vez de mejor calidad y con aportes internacionales relevantes.

Se contrató el sistema Scholastica ${ }^{\circledR}$ para revisión de pares de los manuscritos enviados a la revista. Esto permitió el ingreso online de todos los trabajos, la comunicación con los revisores de manera fluida y mantener un registro de revisores de manera ordenada por especialidad. Esta actividad disciplinada de revisores ha permitido bajar los tiempos promedio de revisión a 16,3 días. Además, se implementó el sistema Cross Ref $^{\circledR}$ para evitar el plagio de artículos, lo 
que quedó establecido en las "Instrucciones a los Autores".

Hubo un aumento del número de ingresos de trabajos en $125 \%$ en comparación del año 2018, aumentando el interés de autores iberoamericanos a enviar sus trabajos. Entre los países más destacados están: Colombia 28\%, Argentina 25\%, Uruguay $18 \%$, México $16 \%$ y España 15\%. Además, aumentó el ingreso de trabajos con temas de pacientes en unidades de cuidado intensivo (30\%), lo que motivó a agregar al comité editorial a un par experto en intensivo, el Dr. Eduardo Kattan (Pontificia Universidad Católica de Chile). Dado a este aumento de ingresos de trabajos la revista se publica de manera bimensual por primera vez desde su fundación.
El número diario de visitas en promedio fluctúa entre 450 y 700 visitas mientras que el Twitter $^{\circledR}$ de la revista chilena (@chileanestesia) tiene alrededor de 750 seguidores. Hay una continua publicación de los trabajos en las redes sociales $\left(\right.$ Facebook $^{\circledR}$, Linkedin $\left.{ }^{\circledR}\right)$, con un aumento de visitas a la página de manera sostenida y con permanencia en ella en promedio de 8,5 minutos.

La revista está más activa y prestigiada que nunca. El objetivo a mediano plazo es que acceda a la indexación propia de las grandes revistas, que es el lugar que nuestra revista merece.

En resumen, estos últimos tres años han sido fructíferos y se ha avanzado de acuerdo a lo planteadeo por el Comité. Es de esperar que el entusiasmo se mantenga o incluso mejore.

\section{Referencias}

1. Ministerio de salud. Subsecretaría de salud pública. Regula los derechos y deberes que tienen las personas en relación con acciones vinculadas a su atención en salud, 2012.

2. Ministerio de salud. Subsecretaría de salud pública. Sobre la investigacion cientifica en el ser humano, su genoma, y prohibe la clonacion humana, 2006.

3. Ministerio secretaría general de la presidencia. Sobre proteccion de la vida privada, 1999.

4. Lacassie HJ, Rolle A, Cortínez LI, Solari S, Corvetto MA, Altermatt FR. Pharmacokinetics of levobupivacaine with epinephrine in transversus abdominis plane block for postoperative analgesia after Caesarean section. Br J Anaesth. 2018 Aug;121(2):469-75. https://doi. org/10.1016/j.bja.2018.02.070 PMID:30032887

5. Aliste J, Layera S, Bravo D, Fernández D, Jara Á, García A, et al. Randomized comparison between perineural dexamethasone and dexmedetomidine for ultrasound-guided infraclavicular block. Regional Anesthesia \& Pain Medicine [Internet]. BMJ; 2019 Jul 11;44(10):911-6. http:// dx.doi.org/10.1136/rapm-2019100680

6. Balkenhol M, Castillo A, Soto M, Feijoo M, Merino W, Balkenhol M. [Measurement of the carbon footprint at a regional hospital]. Rev Med Chil. 2018 Dec;146(12):13849. https://doi.org/10.4067/ s0034-98872018001201384 PMID:30848740

7. Fuentes R, Cortínez LI, Contreras $\mathrm{V}$, Ibacache $\mathrm{M}$, Anderson BJ. Propofol pharmacokinetic and pharmacodynamic profile and its electroencephalographic interaction with remifentanil in children. Paediatr Anaesth. 2018 Dec;28(12):1078-86. https:// doi.org/10.1111/pan.13486 PMID:30307663

8. Aliste J, Bravo D, Finlayson RJ, Tran DQ. A randomized comparison between interscalene and combined infraclavicularsuprascapular blocks for arthroscopic shoulder surgery. Canadian Journal of Anesthesia/ Journal canadien d'anesthésie [Internet]. Springer Science and
Business Media LLC; 2017 Dec 19;65(3):280-7. http://dx.doi. org/10.1007/s12630-017-10480

9. Bravo D, Aliste J, Layera S, Fernández $D$, Leurcharusmee $P$, Samerchua A, et al. A multicenter, randomized comparison between 2, 5, and $8 \mathrm{mg}$ of perineural dexamethasone for ultrasound-guided infraclavicular block. Reg Anesth Pain Med. 2019 Jan;44(1):46-51. https:// doi.org/10.1136/rapm-2018000032 PMID:30640652

10. Aguirre C. MM, Mayanz S. S, Blanch Z. A, Aranibar L. H, Salazar T. A, Roizen G. G, et al. Registro de paro cardiorrespiratorio perioperatorio en un hospital universitario en el período 2006-2017. Revista Médica de Chile. SciELO Agencia Nacional de Investigacion y Desarrollo (ANID); 2019;147(1):34-40. http://dx.doi.org/10.4067/s003498872019000100034

11. Gutiérrez R, Egaña Jl, Sáez I, Reyes F, Briceño C, Venegas M, et al. Intraoperative Low Alpha Power in the Electroencephalogram Is Associated With Postoperative Subsyndromal Delirium. 
Frontiers in Systems Neuroscience. Frontiers Media SA; 2019 Oct 18;13. http://dx.doi. org/10.3389/fnsys.2019.00056

12. Víctor Contreras I. VCl, Ricardo
Fuentes H. RFH, Constanza Barros $\mathrm{P}$. CBP, Osman Casanueva F. OCF, Gabriela Barrueto D. GBD. Implementación de un programa de reciclaje en pabellón. Revista
Chilena de Anestesia. Asociacion de Medicos Anestesiologos de Chile; 2019 May 15;48(2):13640. http://dx.doi.org/10.25237/ revchilanestv48n02.08 